\title{
Un sujet longtemps tabou
}

\section{Jean Martin}

Dr méd., membre de la rédaction

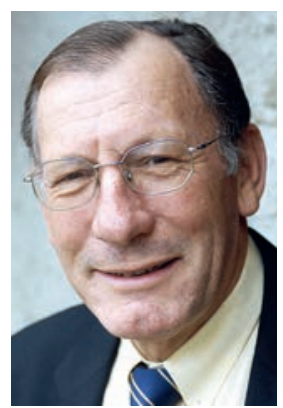

Deux enseignants universitaires publient un gros ouvrage sur le coaching, principalement en milieu hospitalier. Sujet peu évoqué dans le passé mais nous tous avons vécu - ou vivons - des circonstances qui en montrent l'importance. Tant sont monnaie courante dans la vie des professionnels de la santé, médecins mais pas seulement, les difficultés de relations, l'hubris de certains patrons, les compétences insuffisantes de leadership ou de gestion, les antagonismes ou jalousies. Combien de fois a-t-on décrit des séances de commissions distinguées ou de conseils de faculté faisant penser à un jardin d'enfants confus et bruyant... Ou l'histoire d'un chef/une cheffe de services récemment arrivé/e qui fait preuve de peu de psychologie - à l'égard de ses rivaux malheureux par exemple (au reste, le nouveau chef bon dans ses relations, cela existe aussi). Des cas où un professionnel est le souffre-douleur d'un supérieur, voire d'une équipe.

Tout cela est longtemps resté dans le domaine du nondit ou de l'informel, du flou, du délicat. ll est arrivé à beaucoup d'entre nous de chercher à résoudre de telles situations de souffrance, voire de violence. "Au mieux», avec leurs compétences et charisme propres, avec des succès divers.

Les auteurs de «Coaching des soignants» ont été amenés, à l'issue de belles carrières, à accepter des mandats dans de telles situations et à se former en conséquence, retournant sur les «bancs de l'école». Après quarante ans en réanimation/ soins intensifs, le professeur René Chioléro, du CHUV, est ainsi devenu coach certifié, comme Véronique Haynal, psychologue et psychomotricienne à Genève.

Après une présentation de la philosophie du coaching, deux grandes parties. "Les fondamentaux» est la première, traitant de coaching et mentorat (à ne pas confondre), de l'importance de la supervision, du rapport avec la psychothérapie. «La pratique du coaching hospitalier» vient ensuite. Le chapitre 5 par exemple traite des soignants et de leur culture professionnelle: nous ne sommes pas toujours conscients, il s'en faut de beaucoup, de l'importance et des conséquences de cette culture, des socialisations et formatages que nous avons subis et/ou dont nous avons bénéficié. Le chapitre 12 discute le burnout et sa prévention (prendre soin de soi, utilité possible de la "pleine conscience»), le 13 des conflits et de la violence. Enfin, les auteurs décrivent leurs propres parcours et évoquent des perspectives.

Une plus grande présence du coaching dans le système de santé est bienvenue. Il faut à cet égard qu'intervienne un changement d'attitude - même de «cœur»comme celui qu'ont demandé d'autres évolutions: à l'époque, le fait pour un malade de demander un

\section{Une plus grande présence du coaching dans} le système de santé est bienvenue. Il faut qu'intervienne un changement d'attitude à cet égard.

deuxième avis médical était mal ressenti par le médecin traitant, qui y voyait un manque de confiance. Faux! Solliciter un deuxième avis est un souhait logique que le professionnel doit comprendre sans autre. On peut à ce titre aussi rappeler la question de l'accès au dossier médical: certains ont longtemps été réticents à admettre que le cadre légal et éthique prescrit que les patients y ont accès totalement et sans délai. De même, l'éventualité du coaching dans une situation difficile devrait être bien accueillie par tous, y compris de collègues qui s'estiment importants; ceci au vu de son potentiel, mieux qu'avant, de soulager et idéalement régler des problèmes qui ne sont pas rares.

«Coaching des soignants» est un traité pratique, nourri de tableaux et de multiples vignettes tirées de situations vécues $-\mathrm{R}$. Chioléro fait preuve de transparence dans plusieurs cas où il était impliqué. A chaque fois sont décrits la situation de départ, les contacts avec le coach, le mandat, les différentes étapes. Sauf dans une minorité de cas, le résultat n'est cependant pas précisé («happy end», issue difficile, insuccès?). Cela m'a interpelé. Lié au fait que l'accent est mis sur les processus?* Sans que cet ouvrage soit ce que les Anglo-saxons appellent un "page-turner", il vaut la peine de s'y plonger. Il apporte une contribution importante à une meilleure reconnaissance et meilleure pratique du coaching en milieu de soins.

\footnotetext{
A l'ère de MeToo et de mise en évidence de la fréquence des gestes inappropriés à connotation sexuelle dans le domaine médico-hospitalier aussi (il ne fait certainement pas exception), on trouve en tout et pour tout une mention de "remarques sexistes" (p. 342) et une de "sexisme» (p. 382). Etonnant, non? Je m'en suis ouvert au premier auteur qui est lui aussi resté perplexe.
} 